\title{
IMPEDANCE AND THE SINGLE BUNCH LIMIT IN THE APS STORAGE RING
}

\author{
K. C. Harkay ${ }^{+}$, M. Borland, Y.-C. Chae, L. Emery, Z. Huang, E. S. Lesters, ? \\ A. H. Lumpkin, S. V. Milton, N. S. Sereno, B. X. Yang, Advanced Photon Soure 1999 \\ Argonne National Laboratory, Argonne, II 60439 USA
}

\section{Abstract}

The single-bunch current limit and tune shift with current have been documented over time in the 7-GeV Advanced Photon Source (APS) storage ring as a function of lattice, chromaticity, and number of small-gap insertion device (ID) chambers. The contribution to the machine coupling impedance of the 8-mm-gap ID chambers was reported earlier [1]. One 5-mm-gap ID chamber was installed in December 1997. This required changing the lattice to preserve the vertical acceptance. The new lattice reduced the average vertical beta function at the $5-\mathrm{mm}$ chamber as well as at all the other $\mathrm{ID}$ chambers and so has also lowered the effect of the vertical coupling impedance. As additional 8-mm and 5-mm chambers are planned, a more detailed characterization of the impedance is essential. This includes separating the effects of the transitions between the small-gap chambers and the standard chambers from the resistive wall impedance of the small-gap chambers themselves. In this paper, we report on the transverse instabilities and thresholds observed in the vertical and horizontal planes. From these observations, various contributions to the coupling impedance are derived.

\section{INTRODUCTION}

The maximum single-bunch current achieved in the APS storage ring under standard operating conditions is $18 \mathrm{~mA}$. This current limit is most sensitive to the chromaticity, $\xi=\Delta v / \Delta \mathrm{p} / \mathrm{p}$. For these conditions, the chromaticity was $(4,7)$, where the parentheses indicate $\xi_{x}$ and $\xi_{y}$ in that order. The low $\beta_{y}$ lattice installed for use with the 5-mm-gap ID chamber lowers the vertical coupling impedance, but reduces the effectiveness of the chromaticity-correcting sextupoles. The chromaticities are presently about $(1.5,4)$. The single-bunch current limit is subsequently reduced to $-6 \mathrm{~mA}$, determined by the transverse instability threshold.

Typical multibunch user operations require less than 2 $\mathrm{mA}$ per bunch in "triplets" mode, and about $4 \mathrm{~mA}$ /bunch in "singlets" mode. Although present operations are not limited by the single-bunch current limit, additional 8-mm and $5-\mathrm{mm}$ chambers are planned, which will increase the machine coupling impedance. Experimental studies were undertaken to characterize the single-bunch instabilities, and theoretical studies (modeling) are being carried out to determine the impedance model that best fits these data.

-Email: harkay@aps.anl.gov
The instability threshold, growth rate, and current limit were studied as a function of chromaticity, rf voltage (bunch length), if frequency, and orbit position. The growth rate was also measured as a function of current. The instability was monitored using beam position monitor (BPM) history modules, a streak camera, and the tune measurement system.

\section{TUNE SLOPE}

The present complement of twenty 5-m-long small-gap chambers results in tune slopes of -0.0026 per $\mathrm{mA}$ vertically and -0.0008 per $\mathrm{mA}$ horizontally. The vertical tune slope was reduced by $40 \%$ after reducing $\langle\beta\rangle$ in the ID chambers by $60 \%$. Figures 1 and 2 show the vertical and horizontal tune waveforms, respectively, as a function of current. These were obtained by increasing the current in small steps, while using an HP89440A vector signal analyzer to record the beam response to a chirped if signal.

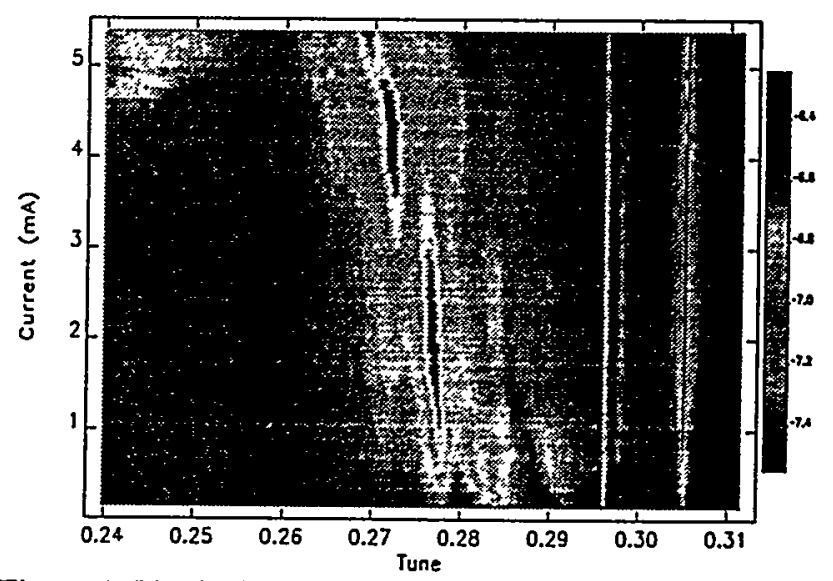

Figure 1: Vertical tune vs. single-bunch current.

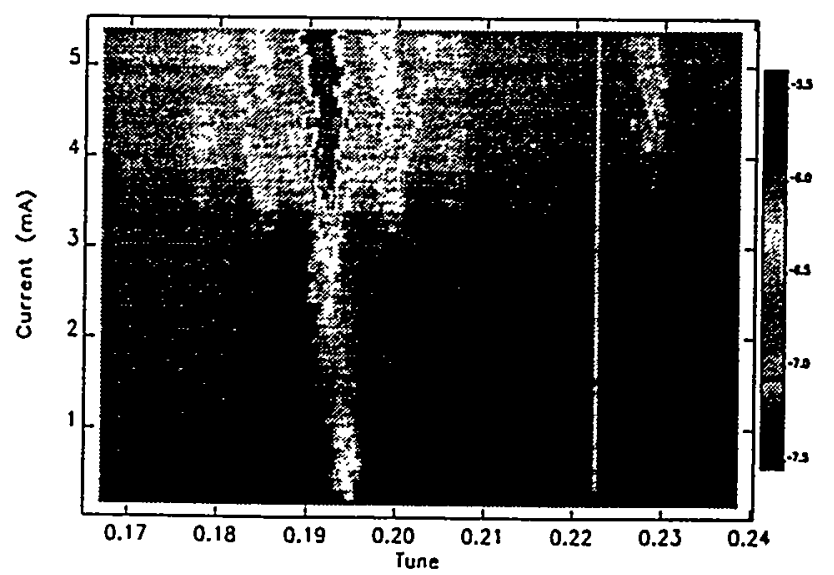

Figure 2: Horizontal tune vs. single-bunch current. 


\section{DISCLAIMER}

This report was prepared as an account of work sponsored by an agency of the United States Government. Neither the United States Government nor any agency thereof, nor any of their employees, make any warranty, express or implied, or assumes any legal liability or responsibility for the accuracy, completeness, or usefulness of any information, apparatus, product, or process disclosed, or represents that its use would not infringe privately owned rights. Reference herein to any specific commercial product, process, or service by trade name, trademark, manufacturer, or otherwise does not necessarily constitute or imply its endorsement, recommendation, or favoring by the United States Government or any agency thereof. The views and opinions of authors expressed herein do not necessarily state or reflect those of the United States Government or any agency thereof. 


\section{DISCLAIMER}

Portions of this document may be illegible in electronic image products. Images are produced from the best available original document. 
The tune waveforms show rich details of the currentdependent beam response. In the vertical plane, the tune (mode 0 is 0.285 at low current) crosses two synchrotron sidebands as the current increases. The synchrotron tune is 0.007 at the nominal if voltage of $9.4 \mathrm{MV}$. The tune appears to just reach mode -3 at the current limit of $5.5 \mathrm{~mA}$. In the horizontal plane, a self-excited tune appears above $3.5 \mathrm{~mA}$. The tune shift over the range is about -0.005 (mode 0 is 0.195 at low current), approximately equal to the synchrotron tune. It appears that the horizontal tune begins to couple to mode -1 near the current limit. At large signal amplitudes, the tune is itself modulated at the synchrotron frequency, which appear as sidebands.

The APS was switched from positron to electron operations in Sept. 1998, and no significant differences in the transverse instability limits were seen.

\section{VERTICAL}

In order to study the transverse planes independently, the vertical chromaticity was reduced while keeping the horizontal almost constant: $(1.0,1.4)$. The single-bunch limit was reduced to $1.9 \mathrm{~mA}$, the lifetime was severely shortened, and the vertical modes 0 and -1 collided. This is consistent with the tune "map" in Fig. 1.

\section{HORIZONTAL}

Observations of the horizontal instability reveal a higher degree of complexity compared to the vertical. It is characterized by a self-excited horizontal tune at a threshold of about $4 \mathrm{~mA}$. Above $4.7 \mathrm{~mA}$ (nominal conditions), a periodic blowup of betatron oscillations of the bunch centroid occurs. This can be seen in Fig. 3, which shows a BPM history recording the $x$-position over 16,000 turns (acquired every turn) with $5.2 \mathrm{~mA}$. The maximum, selflimiting amplitude of the instability is $800 \mu \mathrm{m}$. This is roughly 1.9 times the horizontal rms beam size.

This observation is confirmed using a visible streak camera to image the bending magnet radiation. Figure 4 shows an image acquired in dual-sweep mode. The horizontal beam profile was captured on two time scales: $T_{1}$, which is fast compared to the betatron period, and $T_{2}$, which is slow compared to the instability rise time. Two cycles are seen of the periodic betatron oscillations. Notably, $\sigma_{x}$ does not appear to blow up as the coherent centroid motion decays. Within the spatial resolution, no head-tail motion was detected using streak camera imaging of the $x-t$ plane in synchroscan mode.

The instability growth rate, $1 / \tau_{z}$, was computed as a function of current by fitting the initial blowup in data such as in Fig. 3 , and using $1 / \tau_{\mathrm{rt}}=1 / \tau_{\mathrm{g}}-1 / \tau_{\mathrm{d}}$, where $\tau_{\mathrm{d}}$ is the transverse radiation damping time of $9.46 \mathrm{~ms}$ at 7 $\mathrm{GeV}$. The results are shown in Fig. 5. A preliminary analysis of the amplitude-dependent tune shift was made by performing a fast Fourier transform on the BPM history data in 512-point slices. The horizontal tune peak was fitted to find the frequency. The tune variation with amplitude appears to match a quadratic fit.

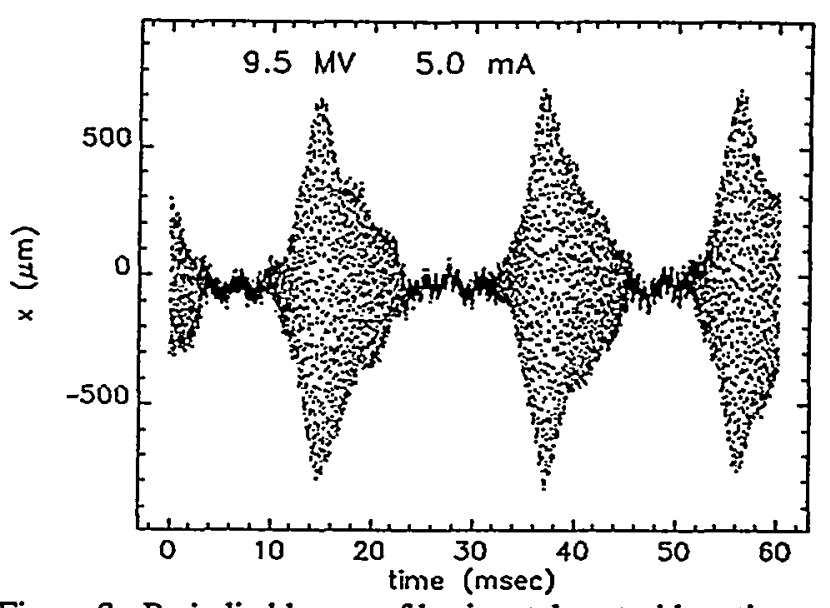

Figure 3: Periodic blowup of horizontal centroid motion.

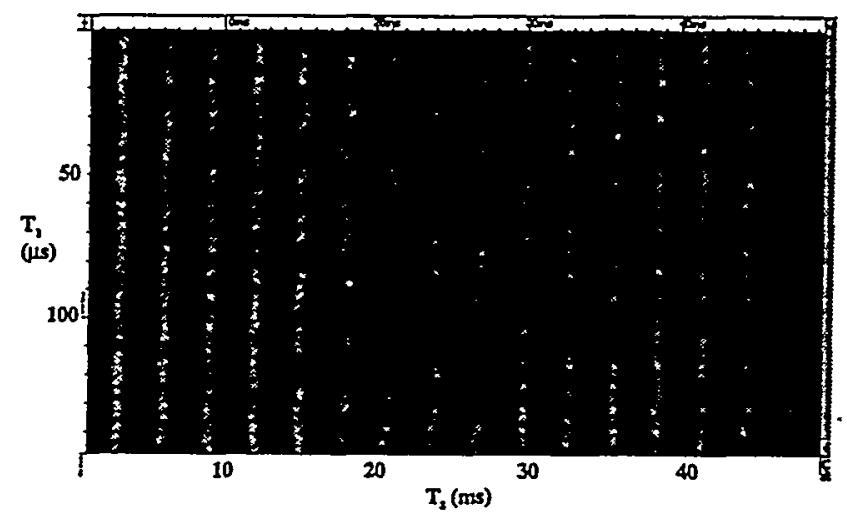

Figure 4: Horizontal oscillations observed using a streak camera in dual-sweep mode.

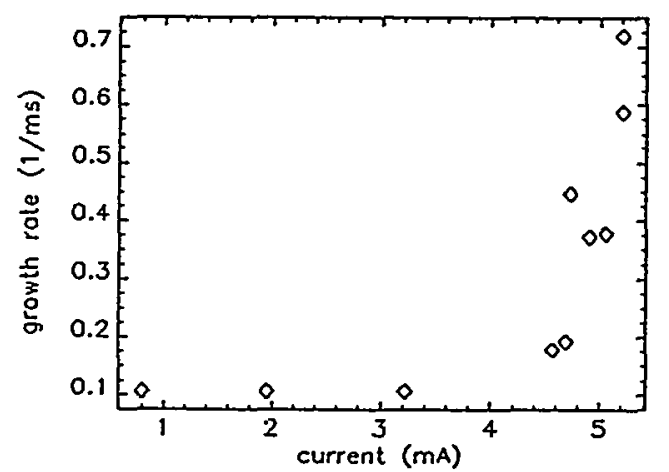

Figure 5: Instability growth rate vs. current.

\subsection{Chromaticity}

At the threshold of $4.7 \mathrm{~mA}$, the periodic instability mode could be stabilized with a $\Delta \xi_{x}$ of 0.6 above nominal. The instability growth rate increased approximately linearly with negative changes in $\xi_{x}$. The instability threshold was not sensitive to even large variations in the tune.

\subsection{Rf voltage}

The if voltage was reduced from $9.5 \mathrm{MV}$ to $7.1 \mathrm{MV}$, resulting in a $20 \%$ increase in the bunch length at $5 \mathrm{~mA}$. The 
current limit increased slightly, from $5.0 \mathrm{~mA}$ at $9.5 \mathrm{MV}$ to $5.5 \mathrm{~mA}$ at $7.9 \mathrm{MV}$ and $5.7 \mathrm{~mA}$ at $7.1 \mathrm{MV}$. More interestingly, with 7.9 MV, the beam underwent periodic blowups around $4.7 \mathrm{~mA}$, but this changed to steady-state, fixedamplitude betatron oscillations at higher currents up to the limit of $5.5 \mathrm{~mA}$ (see Fig. 6). The amplitude of the motion at this limit was about $2 / 3$ of the peak value in the blowup mode. At 7.1 MV, only steady-state oscillations were seen; no blowups were observed at any current up to the limit. In all cases, the threshold for the onset of the selfexcited horizontal tune remained about the same.

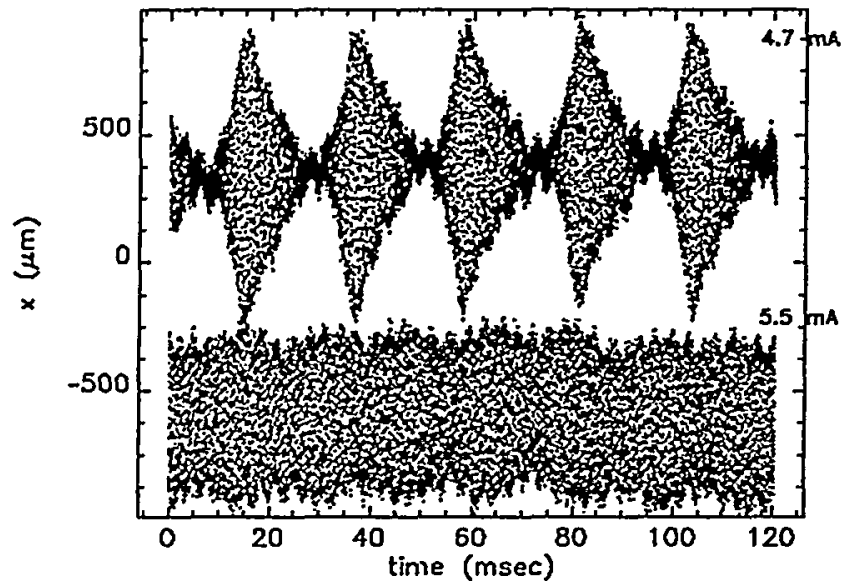

Figure 6: Instability as a function of current at 7.9 MV (plotted offset for clarity).

\subsection{Rffrequency and orbit}

Orbit bumps were used to couple more strongly to the transverse impedance at various places in the ring: 5-mmgap chamber, 19-mm-gap chamber with unique transitions to the standard-aperture chambers, if cavities, and injection region (septum magnet and kicker chambers.) No changes were seen in the instability growth rate for either vertical or horizontal orbit bumps with one exception. A $+1.5 \mathrm{~mm}$ vertical bump in the $5-\mathrm{mm}$ gap chamber stabilized the periodic instability mode horizontally. In addition, changes of $+/-110 \mathrm{~Hz}$ in the rf frequency also stabilized this mode.

\section{SIMULATIONS}

Tracking simulations employed the 6-D tracking program elegant [2]. Resonator impedances are implemented using the following algorithm. A histogram of current density (or its first moment for transverse impedances) is first made. The resonator voltage vs. bin is then obtained by summing the phasor contributions of particles in preceding bins. Particles see only one-half their own induced voltage (fundamental theorem of beam-loading). The voltage is propagated turn-to-turn as a damped sinusoidal oscillation. Tests show excellent agreement with analytical wakefield expressions. elegant includes synchrotron radiation damping and quantum excitation, implemented approximately using the nominal damping decrements and excitation rates.

Simulations of a broad-band resonator (BBR) impedance model $(Q=1)$ reproduced the measured tune slopes, namely, $-0.0008 / \mathrm{mA}$ in the horizontal, and $-0.0026 / \mathrm{mA}$ in the vertical plane. Cutoff frequencies of $5 \mathrm{GHz}$ in the horizontal and $25 \mathrm{GHz}$ in the vertical plane were assumed, determined by the small-gap chambers. The simulations assumed a linear lattice (zero chromaticity) to save computation time. The best fit to the measured values were obtained for a shunt impedance, $R_{s}$, of $0.2 \mathrm{M} \Omega / \mathrm{m}$ in the horizontal plane and $1.2 \mathrm{M} \Omega / \mathrm{m}$ in the vertical plane. The vertical impedance agrees well with the $53 \mathrm{k} \Omega / \mathrm{m}$ per chamber effective impedance value estimated in [1].

A mode-coupling instability (between modes 0 and -1 ) occurs around $4.4 \mathrm{~mA}$ (horizontal) and $2.2 \mathrm{~mA}$ (vertical), nearly reproducing the experimental observations under low chromaticity conditions. Preliminary simulations using a BBR and a fully nonlinear lattice at the nominal chromaticities show some of the instability features, but do not reproduce the periodic blowup seen experimentally.

\section{SUMMARY}

The single-bunch current limit is believed to be dominated by a horizontal instability because of the smaller $\xi_{x}$, compared to $\xi_{y}$. Attempts to raise the instability threshold by raising the chromaticity is hampered by the sextupole current limit. The vertical tune slope $(\alpha<\beta>R$ ) is larger than in the horizontal plane, but the beam is stable to mode -3 with $\xi_{y}=4$. In the horizontal plane, mode -1 is unstable with $\xi_{x}=1.5$. The instability exhibits two modes: periodic blowup and steady-state. The current threshold of the periodic mode decreases with both smaller chromaticity (i.e., tune spread) and shorter bunches (i.e., higher peak current), and the instability growth rate changes linearly with the current. Simulations reproduce the current-dependent tune shift and mode-coupling instability thresholds using a BBR model. The periodic instability mode will be further explored through fully nonlinear simulations. Future machine studies include: additional chromaticity correction, measure the bunch length on the instability time scale, test a single-bunch feedback system and its effect on the instability threshold, and repeat the studies at lower energy.

\section{ACKNOWLEGEMENTS}

The authors would like to thank E. Crosbie for ongoing lattice calculations. This work was supported by the U.S. Department of Energy, Office of Basic Energy Sciences, under Contract No. W-31-109-ENG-38.

\section{REFERENCES}

[1] N. S. Sereno. Y.-C. Chae, K. C. Harkay, A. H. Lumpkin, S. V. Mitton, B. X. Yang, Proc. of 1997 PAC, 1700 (1998)

[2] M. Borland, User's Manual for elegant, v. 12.4, ANL Note LS231 (1995), also www.aps.anl.gov/asd/oag/oagSoftware.shtml 